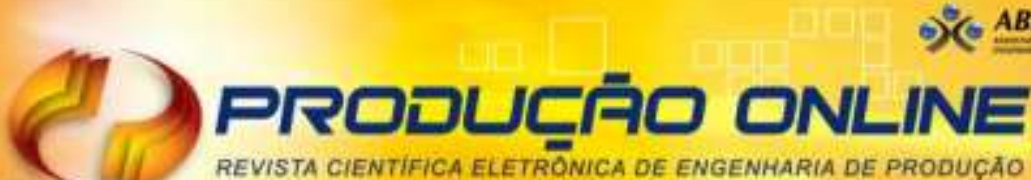 \\ REVISTA CIENTIFICA ELETRÖNICA DE ENGENHARIA DE PRODUÇAO \\ ISSN 1676-1901
}

\section{FAST-FASHION: UMA REVISÃO BIBLIOGRÁFICA SISTEMÁTICA E AGENDA DE PESQUISA}

\section{FAST-FASHION: SYSTEMATIC LITERATURE REVIEW AND RESEARCH AGENDA}

\author{
Lívia Juliana Silva Solino* E-mail: solino.livia@hotmail.com \\ Mario Orestes Aguirre González* E-mail:mario@ct.ufrn.br \\ Maria Emília Morkis Siqueira*E-mail: mariaemiliasiq@gmail.com \\ Williany Ambrosio Nascimento* E-mail: williany.nascimento@gmail.com \\ *Universidade Federal do Rio Grande do Norte (UFRN), Natal, RN
}

Resumo: O fast-fashion (FF) é considerado uma abordagem e fenômeno de influência mundial, que se adequa às necessidades de um mercado exigente e em constante mudança, por ser um modelo de negócios que resulta de uma mistura de rápida fabricação, flexibilidade, baixo custo e abordagens ágeis de varejo. Nesse contexto, a presente pesquisa busca responder, por meio de uma revisão bibliográfica sistemática, como o FF vem sendo abordado nos estudos científicos publicados até o ano de 2013. Mediante a revisão, foi analisado um total de 82 textos, dentre artigos, dissertações e teses. O método de pesquisa utilizado é caracterizado, quanto aos objetivos, como descritiva, e quanto à abordagem como qualitativa e quantitativa. Após realizar a classificação das publicações, foi possível entender o estado da arte, e assim definir um conceito holístico e o termo adequado para o FF. Como também, propor uma agenda de pesquisa com novos enfoques para futuros estudos dentro do tema abordado.

Palavras-chave: Fast-fashion. Revisão bibliográfica sistemática. Agenda de pesquisa.

Abstract: The fast-fashion (FF) is considered an approach and phenomenon of global influence that suit the requirements of a constantly changing and demanding market, since it is a business model resulting of a mixture of fast manufacturing, flexibility, low cost and agile retail approaches. Within this context, this research by a systematic literature review seeks to answer how the FF has been addressed in scientific studies published until the year 2013. Through the review, a total of 82 texts was analysed, among them articles, dissertations and theses. The research method employed is characterized as descriptive, regarding the objectives, and as qualitative and quantitative, on the approach. After conducting the classification of the material, it was possible to understand the state-ofthe-art, and thus set a holistic concept and the appropriate term for the FF. As well as propose a research agenda with new approaches for future studies within the relevant topic.

Keywords: Fast-fashion. Systematic literature review. Research agenda.

\section{INTRODUÇÃO}

Em uma era de grandes mudanças tecnológicas e de abertura do mercado global, a concorrência tornou-se cada vez mais intensa, e isso tem refletido em diversas áreas produtivas, como, por exemplo, na indústria de moda 
contemporânea, que apresenta uma competitividade crescente (Christopher et al., 2004). Por esse ser um sistema extremamente volátil, é imprescindível sempre buscar desenvolver novas estratégias, sendo necessário criar e inovar constantemente para obter vantagens comerciais.

A indústria de moda atualmente se depara com um ambiente onde as preferências dos clientes podem mudar da noite para o dia, o ciclo de vida dos produtos é medido em meses, e o seu valor cai consideravelmente caso seja perdida a última tendência (Sull; Turconi, 2008). O consumidor é o principal responsável por essa transformação, por estar cada vez mais informado e exigente. Dessa maneira, as organizações têm o papel de suprir suas necessidades, saber responder na hora e momento certo, com maior rapidez e flexibilidade.

Para sobreviver neste ambiente surgiram negócios chamados de fast-fashion $(F F)$, os quais têm a capacidade de reagir às tendências do mercado e de melhorar os tempos de resposta. Ele tem suas raízes no desenvolvimento do Quick Response Management (QRM), que de acordo com Suri (2002), é uma estratégia que envolve toda a empresa, muito além de apenas a produção. Porém, para Cietta (2010), Cachon e Swinney (2011), o QRM, apesar de ter prazos de fabricação significativamente curtos, não emprega recursos para obter um design avançado nos produtos, forçando assim a indústria da moda a desenvolver um sistema próprio que emprega simultaneamente as duas características.

A mistura de rápida fabricação, flexibilidade, baixo custo e abordagens ágeis de varejo dentro da mesma organização, resultam no fast-fashion. O mesmo tem alcançado sucesso por conseguir reorganizar a gestão da cadeia têxtil-vestuário em diferentes contextos regionais. Assim como também permite fomentar estratégias de desenvolvimento de uma empresa de integração vertical, tanto em termos de crescimento interno, quanto do gerenciamento de relações externas (Runfola; Guercini, 2013).

Para Cachon e Swinney (2011), o FF não é adequado para todas as indústrias ou todos os produtos. Porém, com a mudança que está acontecendo atualmente, é possível notar que ele não se restringe apenas ao setor têxtil, podendo ser aplicável em outros segmentos que possuem demanda volátil e são sensíveis ao tempo (Cietta, 2010). Visto que comumente o termo "fashion" é associado apenas ao 
vestuário, é importante observar que há um elemento de moda em todas as facetas da vida moderna, podendo ser encontrado até mesmo em alimentos, móveis, ciência, tecnologia, informática, arquitetura, automóveis, artes, artesanato, utensílios de cozinha e de jardinagem (Díaz, 2005; Rickman; Cosenza, 2007; Byun; Sternquist, 2008; Göranson; Hansson, 2010; Cietta, 2010; Düffelmeyer, 2012; Agripina, 2012; Moeng, 2012).

Face ao exposto, é perceptível que características como flexibilidade e rapidez em resposta às mudanças são as bases do FF, aspectos esses de extrema importância para o funcionamento eficiente nas empresas. Porém, o fenômeno é pouco analisado academicamente, fato constatado pela falta de literatura que resuma, estruture ou sintetize o assunto. Desse modo, há uma necessidade de organizar metodicamente os conteúdos estudados (Barnes; Lea-Greenwood, 2006; Göranson; Hansson, 2010; Joy et al., 2012).

Para estruturar um objeto de pesquisa, seu conteúdo e suas variáveis, faz-se necessária uma ampla revisão bibliográfica sobre o mesmo. O método de revisão bibliográfica sistemática gera resultados generalizáveis que podem ser usados para predições razoáveis de eventos futuros em relação ao tema (Webster; Watson, 2002).

Com a finalidade de rever os aspectos relevantes sobre o assunto e mensurar a dimensão na literatura existente, faz-se necessário responder a seguinte problemática: "Como o modelo de negócio denominado fast-fashion vem sendo abordado nos estudos científicos publicados até o ano de 2013?". Objetivando responder o questionamento, realizou-se um levantamento bibliográfico no período determinado para atingir dois propósitos: i) identificar e classificar as publicações por ano, journal, universidade, área, país, foco da pesquisa, método, tipo de abordagem e objetivo; e ii) analisar o estado da arte.

Para explorar as discussões apresentadas acima, o artigo apresenta a seguinte sequência. Além desta seção de caráter introdutório, a segunda parte retrata o método e sistematização de pesquisa adotada. A terceira seção classifica os artigos, dissertações e teses selecionadas, e a quarta analisa o estado da arte da temática em questão. $\mathrm{Na}$ quinta parte é apresentada uma seção com 
recomendações de pesquisas futuras. Por fim, a sexta parte apresenta as considerações finais.

\section{MÉTODO DE PESQUISA}

O artigo pode ser classificado, quanto aos seus objetivos, como descritivo, pois permite expor a temática FF, descrever o cenário, e propor novas linhas de estudo (Yin, 2001). Com relação ao gênero pode ser considerado como teóricoconceitual, uma vez que no tocante aos procedimentos técnicos aplicados, é categorizado como revisão bibliográfica sistemática.

Identificar e organizar os conceitos relevantes sobre um determinado conteúdo é o foco principal da revisão da literatura (Rowley; Slack, 2004). A revisão bibliográfica sistemática, por sua vez, é uma abordagem de pesquisa confiável, visto que é abrangente e apresenta explicitamente os meios utilizados e os resultados obtidos (Pai et al., 2004), além de incluir uma busca minuciosa das publicações e a possibilidade de replicação do método (Cook et al., 1997).

A pesquisa quantitativa garante aos dados coletados precisão através do tratamento numérico e estatístico, evitando distorções de análise. Enquanto que a qualitativa, por sua vez, busca estabelecer sentido e significação ao objeto de estudo. Dessa forma, a abordagem científica utilizada é considerada qualitativa e quantitativa (Creswell, 2007), tendo em vista que o levantamento e avaliação dos textos requereu tratamento tanto objetivo quanto subjetivo.

Um processo de sete etapas, adaptado de González e Toledo (2012), foi adotado na condução deste trabalho para selecionar e recuperar documentos: (i) seleção de base de dados informatizadas, (ii) identificação da palavra-chave, (iii) busca dos documentos, (iv) revisão manual dos resumos, (v) primeira exclusão dos textos, (vi) revisão dos textos completos selecionados, e (vii) segunda exclusão dos textos. Nos tópicos seguintes serão discutidos os principais passos na condução da revisão bibliográfica sistemática no que se refere ao procedimento de pesquisa, e, em seguida, será feita a classificação da literatura sobre FF quanto ao tempo, fontes, e metodologia. 


\subsection{Procedimento da pesquisa}

A presente pesquisa foi conduzida em sete etapas. Primeiramente, (i) foram selecionadas três bases de dados informatizadas: portal Periódicos CAPES (Coordenação de Aperfeiçoamento de Pessoal de Nível Superior), Web of Science, e Google Acadêmico. Em seguida, para que fosse feita a filtragem dos textos, (ii) foi definida a palavra-chave desta revisão: fast-fashion. (iii) As buscas procederam de maneira diversa a fim de conseguir um número mais expressivo de publicações. No portal Periódico CAPES aconteceu através do uso da ferramenta Metabusca, onde se utilizou a palavra-chave, e foi aplicada a ferramenta do filtro para restringir a procura em artigos científicos. Na Web of Science foram usados os mesmos parâmetros do portal CAPES. Por fim, no Google Acadêmico foram empregadas as palavras-chave fast-fashion e thesis, na intenção de obter tanto artigos científicos quanto teses e dissertações. Não foi utilizado filtro para limitar os anos da busca, sendo a filtragem feita até o ano de 2013.

Após (iv) verificação dos resumos, foram selecionados 105 textos. Em seguida foi feita a aplicação do primeiro filtro de exclusão, (v) onde se eliminou todos os documentos que não disponibilizaram o material completo, reduzindo o montante para 97. Na sexta etapa, a partir da (vi) leitura completa dos 97 trabalhos, realizou-se a classificação dos mesmos quanto ao seu conteúdo mediante fichamento, e por meio deste foi possível reunir os dados de modo a compreendê-los melhor.

A construção da base de dados levou em consideração a problemática do artigo, que procura entender como o fast-fashion vem sendo abordado nos estudos científicos. Desta forma foram listados os principais pontos que deveriam ser observados durante a leitura. Podem-se dividir estes pontos em classificação da literatura, características do FF e tópicos específicos. Os itens que correspondem à classificação da literatura devem oferecer informações que agrupem quanto à fonte, à metodologia, e ao comportamento ao longo do tempo. Os pontos a serem observados no exame dos textos que se referem às características, procuram trazer informações sobre os conceitos existentes na literatura, o termo utilizado para denominar o fast-fashion, a área funcional a que pertence, os objetivos de desempenho relacionados, e sobre quais variáveis foram tratadas pelos autores. Para completar a lista de itens, buscou-se saber se o FF estava sendo aplicado em Revista Produção Online, Florianópolis, SC, v.15, n. 3, p. 1021-1048, jul./set. 2015. 
outros setores, e se havia alguma outra denominação para a expressão. Assim, com a base de dados estruturada, realizou-se a leitura dos documentos e o preenchimento da mesma.

$\mathrm{Na}$ etapa seguinte (vii) ocorreu a aplicação do segundo filtro de exclusão, onde foram retirados os textos que apenas citaram em algum momento o termo fastfashion, não tratando de temáticas relevantes ao estudo. Posterior a aplicação deste filtro, 82 trabalhos foram considerados significativos para as discussões sobre FF. De acordo com Fonseca e Rozenfeld (2012) por este método não propor que se faça uma pesquisa cruzada, podem existir trabalhos que referenciam outros autores e que merecem atenção do pesquisador. Assim, outros trabalhos citados nos estudos primários podem ser incluídos para análise posterior.

\section{CLASSIFICAÇÃO DOS ARTIGOS ANALISADOS}

Uma visão geral da literatura pode ser feita a partir da análise da mesma em relação aos: anos de publicação, fontes dos trabalhos, tipo de pesquisa, local de residência do autor e da sede do objeto de estudo.

\subsection{Literatura ao longo do tempo}

Estudos contendo o termo fast-fashion começaram a surgir no ano de 2005. O número de publicações durante os cinco primeiros anos (2005-2009) apresentouse de maneira sutil. Observando a figura 1, é possível perceber uma tendência de crescimento a partir de 2010.

Revista Produção Online, Florianópolis, SC, v.15, n. 3, p. 1021-1048, jul./set. 2015. 
Figura 1 - Distribuição das publicações ao longo do tempo

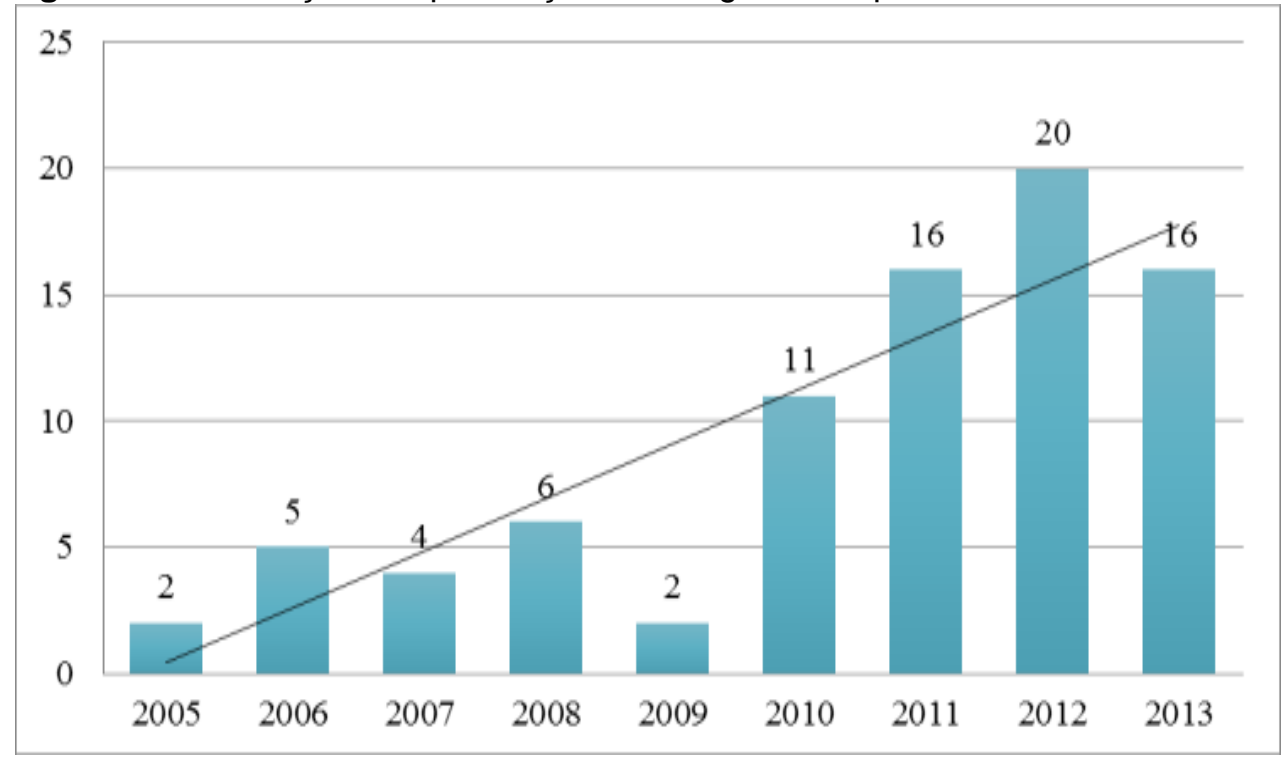

Fonte: Elaborado pelos autores (2014)

No primeiro período (2005-2009), os dados comportam-se de maneira instável, apresentando uma média aproximada de 4 publicações por ano. Entretanto, no segundo conjunto (2010-2013), a média aproximada de trabalhos subiu para 16, sendo em 2012 seu auge, com 20 publicações.

\subsection{Literatura e suas fontes}

Dos 82 textos considerados relevantes para a revisão bibliográfica sistemática sobre o tema, 48 deles são artigos publicados em periódicos internacionais e 34 são dissertações de mestrado e teses de doutorado. Analisando a distribuição de artigos em periódicos (tabela 1), percebe-se que apenas dois deles possuem $31,3 \%$ das publicações, sendo eles Journal of Fashion Marketing and Management e Fashion Theory, enquanto $47,9 \%$ dos artigos são de 23 diferentes fontes. 
Tabela 1 - Distribuição de artigos em periódicos

\begin{tabular}{lcc}
\hline \multicolumn{1}{c}{ Periódico } & Quantidade & Porcentagem \\
\hline Journal of Fashion Marketing and Management & 11 & $22,9 \%$ \\
Fashion Theory & 4 & $8,3 \%$ \\
International Handbooks on Information Systems & 2 & $4,2 \%$ \\
International Journal of Retail \& Distribution Management & 2 & $4,2 \%$ \\
Management Science & 2 & $4,2 \%$ \\
Operations Research & 2 & $4,2 \%$ \\
The International Review of Retail, Distribution and Consumer Research & 2 & $4,2 \%$ \\
Outros & 23 & $47,9 \%$ \\
\hline
\end{tabular}

Fonte: Elaborado pelos autores (2014)

Com relação aos trabalhos provenientes da conclusão de mestrados e doutorados (tabela 2), 38,2\% são referentes a três universidades. O maior número de pesquisas foi encontrado nas faculdades/universidades: The Swedish School of Textiles (Suécia), Massachusetts Institute of Technology (Estados Unidos) e Jönköping University (Suécia). Dos 21 textos restantes, cada um é proveniente de um instituto distinto.

Tabela 2 - Distribuição de trabalhos por universidade

\begin{tabular}{lcc}
\hline \multicolumn{1}{c}{ Universidade } & Quantidade & Porcentagem \\
\hline The Swedish School of Textiles & 6 & $17,6 \%$ \\
Massachusetts Institute of Technology & 5 & $14,7 \%$ \\
Jönköping University & 2 & $5,9 \%$ \\
Outros & 21 & $61,8 \%$ \\
\hline
\end{tabular}

Fonte: Elaborada pelos autores (2014)

No tocante ao programa de pós-graduação onde se realizou o estudo, uma grande diversidade foi encontrada, sendo a maioria (seis estudos) proveniente do curso Fashion Management, e destes, dois são especializações em Fashion Marketing and Retailing. Outras publicações foram resultado da conclusão de Business Administration e de Science, e outras de International Logistics and Supply Chain Management, International Marketing e Doctor of Philosophy, cursos que possuem dois trabalhos cada um.

\subsection{Literatura por tipo de pesquisa, residência do autor e sede do objeto de estudo}

De acordo com Rowley e Slack (2004), a revisão da literatura identifica e organiza as características relevantes, resumindo assim o estado da arte do tema Revista Produção Online, Florianópolis, SC, v.15, n. 3, p. 1021-1048, jul./set. 2015. 
estudado. Para a análise da classificação dos textos foram considerados os seguintes pontos principais: método; abordagem; objetivos; objeto; foco do autor; país sede; país foco; e empresa estudada.

Quanto ao Método de pesquisa utilizado, o Estudo de Caso apresentou-se como predominante em $46 \%$ dos trabalhos seguidos por Survey (25\%), Grupo Focal e Revisão da Literatura, ambos com 11\%.

No que se refere à Abordagem, a maior parte dos textos foram classificados como Qualitativos com 52\% do total, seguido pela abordagem Quali-Quantitativa (28\%), Quantitativa, presente em 11\%, e por fim, a Quanti-Qualitativa (9\%).

Com relação aos Objetivos, as classificações Exploratória, Descritiva e Explicativa, obtiveram 55\%, 33\% e 12\%, respectivamente. No tocante ao Objeto, a Pesquisa de Campo alcançou uma abrangência de $79 \%$ dos textos seguidas pela Pesquisa Bibliográfica (19\%), e a Pesquisa de Laboratório (2\%).

No que concerne a procedência do Autor, tem-se a seguinte ordem de participação, Universidade (93\%), Empresa (4\%), Centro de Pesquisa (2\%) e Outros $(1 \%)$.

A figura 2 expõe o resultado da averiguação do país sede de cada instituição de ensino dos autores que desenvolveram as pesquisas. Os Estados Unidos apresentam-se em primeiro lugar, com 30 estudos, seguido da Suécia (18), Reino Unido (13) e Itália (8). 
Figura 2 - Distribuição dos artigos por país sede dos autores

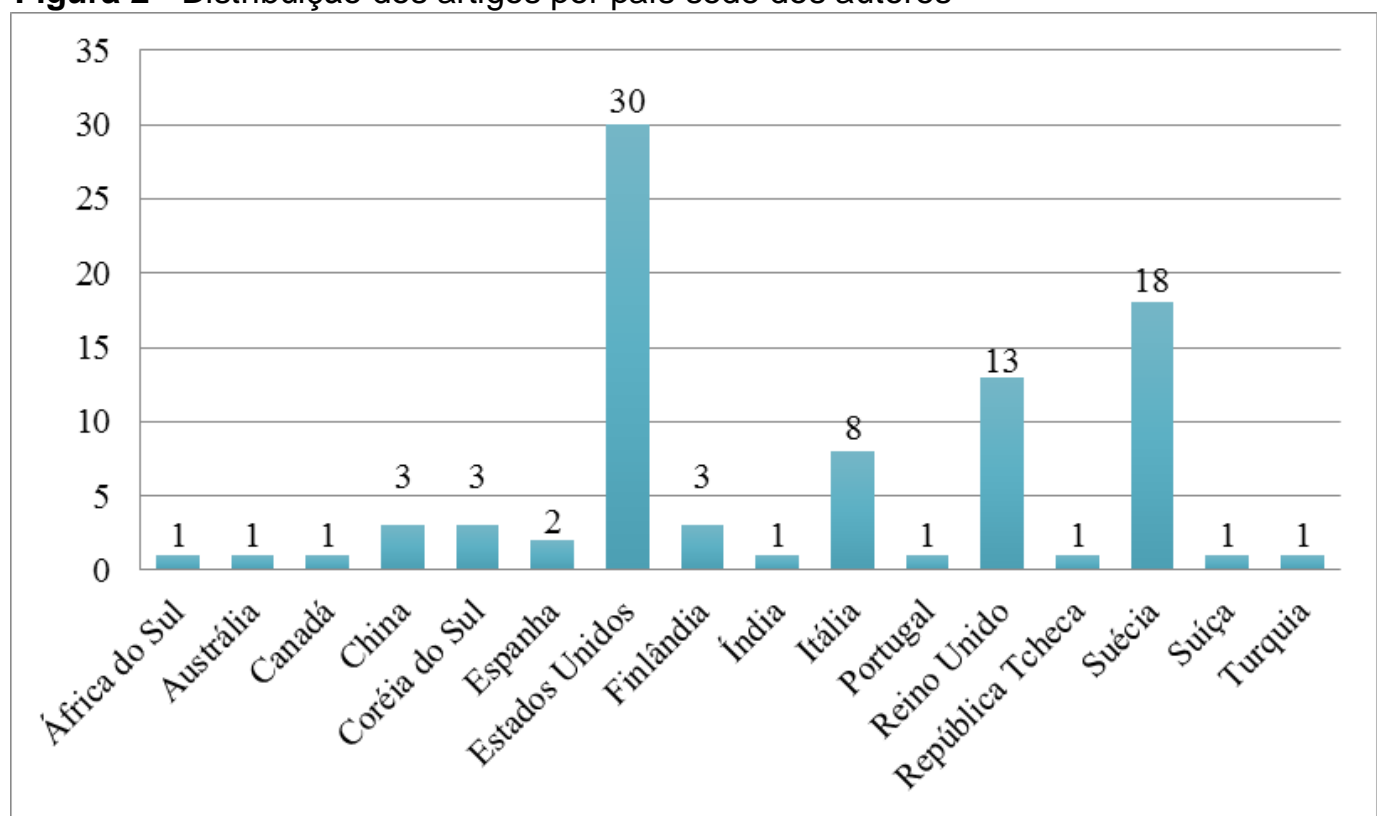

Fonte: Elaborado pelos autores (2014)

Considerando o país foco do estudo, isto é, o local que foi analisado, seja em relação à empresa ou ao mercado consumidor, o Reino Unido aparece em 11 pesquisas, seguido pela Suécia e Estados Unidos (10), Itália (7), China (6) e Turquia (4) - figura 3.

Realizando uma análise conjunta de ambos os gráficos apresentados, verifica-se que em $78 \%$ dos estudos, pelo menos um autor pesquisador pertence a um instituto localizado no país foco da pesquisa.

Da população das publicações estudadas, 48 tiveram sua análise baseada em uma empresa do ramo têxtil. A Zara, empresa espanhola referência em obter sucesso aplicando o FF, foi estudada em $34 \%$ dos textos analisados, seguido pela Hennes \& Mauritz - H\&M (Suécia) e da Gina Tricot (Suécia), com 17\% e 5\%, respectivamente. 
Figura 3 - Distribuição dos artigos por país do objeto de estudo

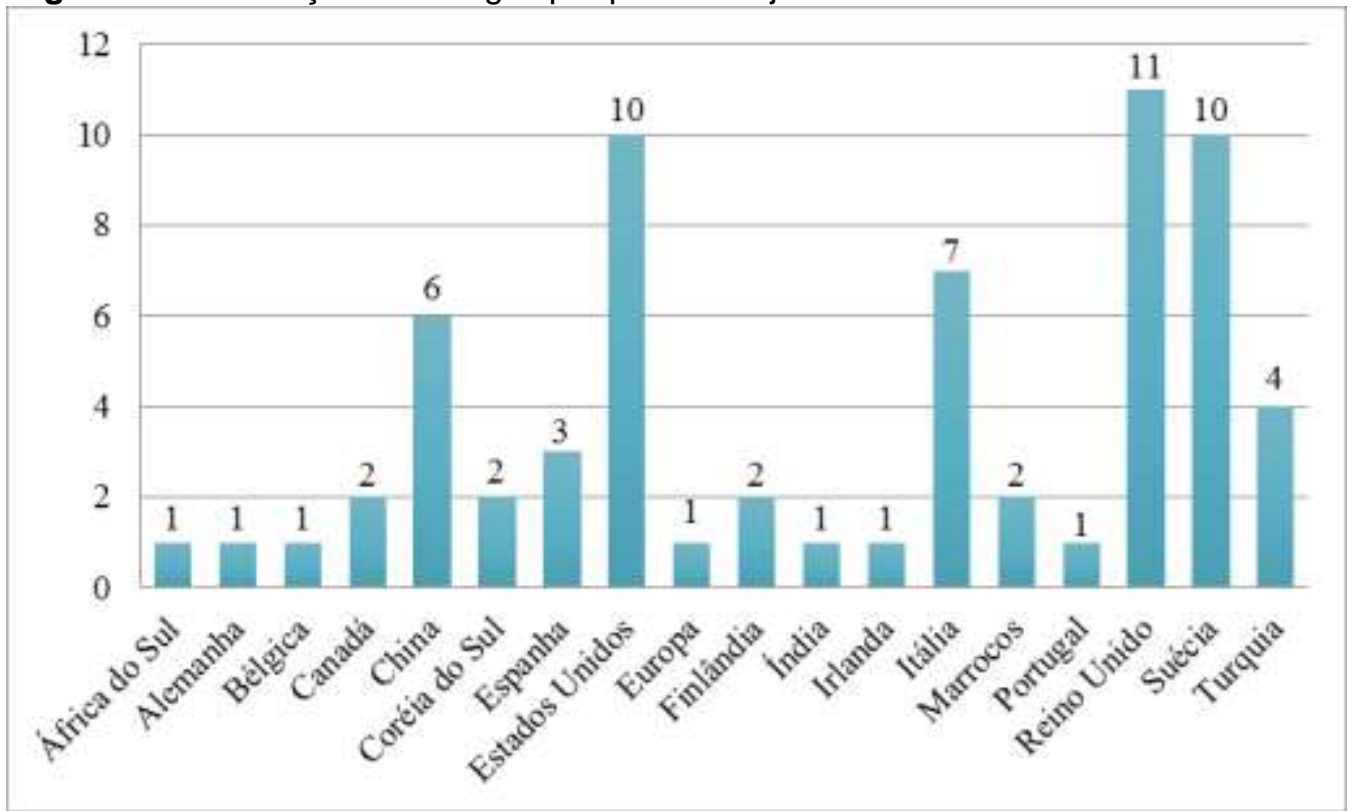

Fonte: Elaborado pelos autores (2014)

Se for feita uma comparação analisando apenas os estudos realizados em empresas (tabela 3), percebe-se que $88 \%$ do foco da pesquisa são provenientes de Universidades, na sequência Empresas (6\%), Centros de pesquisa (4\%), e Outros $(2 \%)$.

Tabela 3 - Comparação foco da pesquisa para estudos em empresas

\section{Foco da}

pesquisa Quantidade Porcentagem

\begin{tabular}{lcc}
\hline Universidade & 44 & $88 \%$ \\
Empresa & 3 & $6 \%$ \\
Centro de & 2 & $4 \%$ \\
Pesquisa & $2 \%$ \\
Foco Teórico & 1 & $2 \%$ \\
\hline \multicolumn{2}{l}{ Fonte: Elaborada pelos autores $(2014)$}
\end{tabular}

Fonte: Elaborada pelos autores (2014)

Ainda analisando os estudos realizados em empresas, $44 \%$ utilizaram apenas uma empresa para basear seu estudo, $27 \%$ duas organizações e $15 \%$ três.

Revista Produção Online, Florianópolis, SC, v.15, n. 3, p. 1021-1048, jul./set. 2015. 


\section{ESTADO DA ARTE SOBRE O FAST-FASHION}

Tópicos relevantes que despontam na literatura sobre FF podem ser analisados nos tópicos: área funcional considerada no estudo, definição conceitual, termo utilizado pelos pesquisadores, outras denominações para o FF, e objetivos de desempenho no FF.

\section{1 Área funcional e objetivos de desempenho considerados no estudo}

Slack et al. (2013) separa as áreas funcionais de uma empresa em dois grupos: as funções centrais e as funções de apoio. Com relação às centrais, elas se subdividem em: marketing, responsável pela comunicação dos produtos da organização para seus mercados; desenvolvimento de produtos, responsável pelo desenvolvimento e modificação de bens e serviços; e, produção, responsável pela realização/entrega dos pedidos. Relativamente às funções de apoio, as quais dão suporte para que as outras funcionem efetivamente, têm-se duas subdivisões: contábil-financeira, a qual fornece informações para tomada de decisões e gerencia os recursos financeiros; e, recursos humanos, que recruta/desenvolve os funcionários.

Ao considerar as informações presentes na figura 4, nota-se que a função produção é a mais estudada, estando presente em $42 \%$ das publicações analisadas, seguido pelo marketing (38\%), desenvolvimento de produtos (9\%), uma análise geral da organização (5\%), e depois as funções de apoio, contábil-financeira (3\%) e recursos humanos $(2 \%)$. 
Figura 4 - Classificação quanto à área funcional

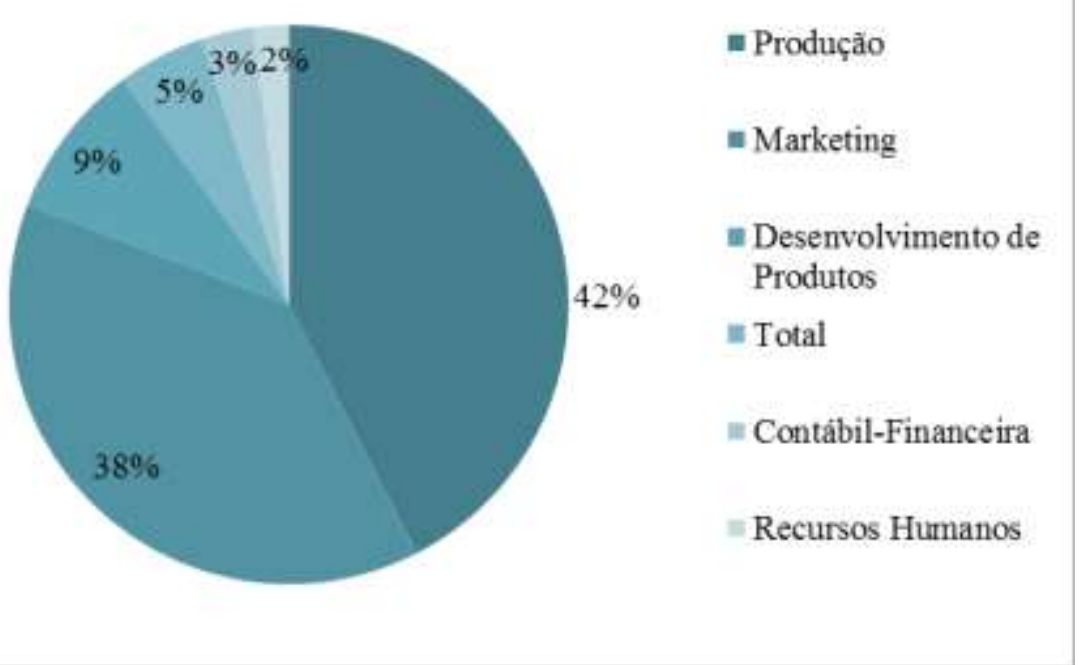

Fonte: Elaborado pelos autores (2014)

Por fim, tem-se a análise das Variáveis de Objetivos de Desempenho analisadas em relação à definição que cada autor assumiu do conceito fast-fashion. O ponto principal determinado foi a Rapidez $(45,1 \%)$, seguida pela Flexibilidade (25\%), como pode ser observado na tabela 4.

Tabela 4 - Variáveis objetivos de desempenho

\begin{tabular}{lc}
\hline \multicolumn{1}{c}{$\begin{array}{c}\text { Objetivos de } \\
\text { desempenho }\end{array}$} & Porcentagem \\
\hline Rapidez & $45,1 \%$ \\
Flexibilidade & $25,0 \%$ \\
Custo & $17,7 \%$ \\
Confiabilidade de entrega & $8,5 \%$ \\
Qualidade & $3,7 \%$ \\
\hline
\end{tabular}

Fonte: Elaboração própria (2014)

Nos textos analisados, pode-se observar que a maioria das organizações com o modelo fast-fashion presente, unem os objetivos de desempenho a fim de otimizar cada vez mais o processo produtivo. Dos trabalhos, 59\% assumem como importante a utilização de dois objetivos de desempenho, apenas um (19\%), três (16\%), e quatro (5\%). 


\subsection{Definição conceitual do fast-fashion}

Mediante a análise das publicações, encontrou-se 74 textos que conceituam o fast-fashion, nesses foram obtidos 33 diferentes interpretações, que podem ser justificadas, por ser um assunto relativamente novo e mesmo sendo fortemente priorizado no setor de negócios e mídia, na academia ainda é pequena a atenção. Desse modo, há pouco material compartilhado, e isso faz com que o conceito não tenha sido consolidado até o momento (Göranson; Hansson, 2010; Cietta, 2010; Joy et al., 2012).

No ano de 2013 nenhum novo conceito foi encontrado, no período de 2001 até 2012 o desenvolvimento e a evolução conceitual aconteceram de forma relativamente lenta, onde a média de novas definições era em cerca de duas por ano. Porém, com exceção de 2004 e 2006, quando foram publicadas seis, em ambos os períodos. E em seguida, tem-se o ano de $2010 \mathrm{com}$ cinco. Frente ao estudo, é possível afirmar que ao decorrer do tempo poucos autores criaram sua própria definição.

Depois de listados todos os conceitos, foram selecionados os dez mais relevantes para estudo. A tabela 5 visa expor as principais definições de diferentes autores sobre o FF. Os mais citados foram os conceitos de Barnes e Lea-Greenwood (2006; 2010), professoras do Department of Clothing Design and Technology da Manchester Metropolitan University (Reino Unido), que está presente em dez publicações. Posteriormente, Cachon e Swinney (2011) citado em seis publicações, Cachon é professor da Wharton School - University of Pennsylvania, e Swinney é professor no grupo de Operations Management da Duke University Fuqua School of Business (Estados Unidos da América). Em seguida têm-se Sull e Turconi (2008), professores da London Business School (Reino Unido), citado em cinco publicações. 
Tabela 5 - Conceitos do fast-fashion

\begin{tabular}{|c|c|c|}
\hline Autor & Conceito & Referência \\
\hline $\begin{array}{l}\text { Barnes; Lea- } \\
\text { Greenwood, } \\
2006 / 10\end{array}$ & $\begin{array}{l}\text { Uma estratégia de negócios que visa diminuir os } \\
\text { processos que estão envolvidos no ciclo de compra e } \\
\text { reduzir os prazos de entrega das ordens de compra } \\
\text { até que o seu novo produto de design de moda chega } \\
\text { às lojas, a fim de atender a demanda do consumidor } \\
\text { durante a alta temporada ou quaisquer outras } \\
\text { estações. }\end{array}$ & $\begin{array}{l}\text { Barnes; Lea-Greenwood, } \\
\text { 2010; Cheng, 2010; } \\
\text { Göranson; Hansson, } \\
\text { 2010; Choudhury, 2011; } \\
\text { Fojtu, 2011; Hansson, } \\
\text { 2011; Memic; Minhas, } \\
\text { 2011; Kim; Park, 2013; } \\
\text { Räisänen, 2013; Runfola; } \\
\text { Guercini. 2013 }\end{array}$ \\
\hline $\begin{array}{c}\text { Cachon; } \\
\text { Swiney, } 2011\end{array}$ & $\begin{array}{l}\text { Um sistema fast-fashion combina pelo menos dois } \\
\text { componentes: (i) curtos prazos de produção e } \\
\text { distribuição, permitindo uma aproximação da oferta } \\
\text { com a demanda incerta (ao qual nos referimos como } \\
\text { técnicas de quick response); (ii) design de produto } \\
\text { muito na moda ("trendy") (que se referem a técnicas de } \\
\text { design avançado). }\end{array}$ & $\begin{array}{l}\text { Cachon; Swinney, 2011; } \\
\text { Agripina, 2012; Bonnefoi, } \\
\text { 2012; Carrion; Caceres, } \\
\text { 2012; Gabrielli et al., } \\
\text { 2013; Miller, } 2013\end{array}$ \\
\hline $\begin{array}{c}\text { Sull; Turconi, } \\
2008\end{array}$ & $\begin{array}{l}\text { Fast-fashion descreve a estratégia de varejo de } \\
\text { adaptar sortimentos de mercadorias com as } \\
\text { tendências atuais e emergentes tão rápida e } \\
\text { eficazmente quanto possível. }\end{array}$ & $\begin{array}{l}\text { Sull; Turconi, } 2008 ; \\
\text { Göranson; } \quad \text { Hansson, } \\
2010 ; \quad \text { Garro, 2011; } \\
\text { Chatvijit, 2012; Portway, } \\
2012\end{array}$ \\
\hline $\begin{array}{r}\text { Bruce } \\
2004\end{array}$ & $\begin{array}{l}\text { O aumento da complexidade e os custos são } \\
\text { particularmente importantes para a indústria de varejo } \\
\text { de vestuário em massa, onde os ciclos de vida } \\
\text { extremamente curtos - altos níveis de produtos } \\
\text { personalizados, sazonalidade e demanda volátil } \\
\text { exigem operações de armazém eficazes. }\end{array}$ & $\begin{array}{l}\text { Hilletofth; Hilmola, 2008; } \\
\text { Bruce; Daly, 2011; } \\
\text { Cagliano et al., 2011; } \\
\text { Brandting; Johansson, } \\
2012\end{array}$ \\
\hline $\begin{array}{c}\text { Caro; Gallien } \\
2010 / 12\end{array}$ & $\begin{array}{l}\text { Estratégia operacional de varejistas de fast-fashion } \\
\text { consiste em oferecer ao longo da temporada de } \\
\text { vendas um grande número de artigos diferentes, cada } \\
\text { vez mais modernos, com um ciclo de vida } \\
\text { relativamente curto de apenas algumas semanas. }\end{array}$ & $\begin{array}{l}\text { Caro; Gallien, 2010; Caro; } \\
\text { Martínez-De-Albeniz, } \\
\text { 2010; Caro et al., 2010; } \\
\text { Caro; Gallien, } 2012\end{array}$ \\
\hline $\begin{array}{l}\text { Doer } \\
\text { Crear }\end{array}$ & $\begin{array}{l}\text { A capacidade de monitorar as preferências dos } \\
\text { consumidores de forma rápida e para identificar novos } \\
\text { projetos potencialmente populares pela proximidade } \\
\text { diária para os mercados de moda, imagens de moda e } \\
\text { fabricantes de moda. Moda de rua não é projetada na } \\
\text { forma tradicional da alta-costura, mas sim é uma } \\
\text { adaptação de projetos existentes e produzidas em } \\
\text { diferentes tipos de materiais, cores e silhuetas. }\end{array}$ & $\begin{array}{l}\text { Doeringer; Crean, 2006; } \\
\text { Choi et al., 2011; Kim; } \\
\text { Park, 2013 }\end{array}$ \\
\hline $\begin{array}{l}\text { Fernie et al., } \\
\quad 2004\end{array}$ & $\begin{array}{l}\text { Fast-fashion é o termo usado para indicar as } \\
\text { estratégias adotadas pelos varejistas de moda para } \\
\text { refletir as tendências de moda atuais e emergentes } \\
\text { com rapidez e eficácia em sortimentos de mercadorias } \\
\text { atuais. }\end{array}$ & $\begin{array}{l}\text { Sheridan et al., 2006; } \\
\text { Byun; Sternquist, 2008; } \\
\text { Madhani, } 2011\end{array}$ \\
\hline Muran, 2007 & $\begin{array}{l}\text { varejistas de moda rápida apresentam coleções de } \\
\text { pas com base nas últimas tendências da moda, } \\
\text { s projetados e fabricados de forma rápida e barata, } \\
\text { a permitir que o consumidor convencional aproveite } \\
\text { los de roupas atuais a preços mais baixos. }\end{array}$ & $\begin{array}{ll}\text { Ka Yu; Zastezhko, 2008; } & \text { 2012; } \\
\text { Düffelmeyer, } & \text { 2012 }\end{array}$ \\
\hline
\end{tabular}

Revista Produção Online, Florianópolis, SC, v.15, n. 3, p. 1021-1048, jul./set. 2015. 
Tabela 5 - Conceitos do fast-fashion

\begin{tabular}{|c|c|c|}
\hline Autor & Conceito & Referência \\
\hline $\begin{array}{l}\text { Reinach, } \\
2005\end{array}$ & $\begin{array}{l}\text { Fast-fashion copia as tendências mais atraentes e } \\
\text { promissoras vistas em desfiles de moda, que agora } \\
\text { estão disponíveis em tempo real na internet, graças à } \\
\text { tecnologia da fotografia digital e transformam em } \\
\text { produtos que podem ser colocados no mercado } \\
\text { imediatamente, libertando os lojistas e consumidores } \\
\text { da armadilha da coleção sazonal. Em outras palavras, } \\
\text { fast-fashion serve tendências à la carte. }\end{array}$ & $\begin{array}{l}\text { Reinach, 2005; Tokatli, } \\
\text { 2008; Tokatli et al., } 2010\end{array}$ \\
\hline $\begin{array}{l}\text { Sheridan et } \\
\text { al., } 2006\end{array}$ & $\begin{array}{l}\text { Gira em torno de oferecer as últimas roupas tão rápida } \\
\text { e eficazmente quanto possível, a um preço acessível, } \\
\text { através da combinação de processos rápidos. }\end{array}$ & $\begin{array}{l}\text { Arrigo, 2013; Kim et al., } \\
\text { 2013; Miller, } 2013\end{array}$ \\
\hline
\end{tabular}

Fonte: Elaborada pelos autores (2014)

Considerando as definições encontradas, pode-se concluir que o fast-fashion consiste em um modelo de negócios que consegue unir a estratégia produtiva do quick-response com a utilização de recursos avançados para o design de produtos. Apresenta flexibilidade em todos os setores da cadeia produtiva para que, dessa forma, possa otimizar o processo produtivo e atender com inovação a demanda dos consumidores que possuem gostos frequentemente mutáveis. Além de oferecer tendências atuais de maneira rápida e eficaz na forma de produtos com curto ciclo de vida e baixo custo.

\subsection{Termo utilizado pelos pesquisadores ao citar o fast-fashion}

Foram encontrados diversos termos com a finalidade de classificar o FF, pois ainda não há um consenso se o mesmo é um sistema, um modelo, uma estratégia ou outros. Dentre os 82 textos, 64 mencionaram algum termo. Sendo notória a relação entre o termo adotado pelo autor e a conceituação envolvida em seu estudo.

O termo Estratégia foi encontrado em 42\% dos textos. Mintzberg (2010) defende que a Estratégia não possui um único significado, com base nisso define-a como um plano, um guia ou curso de ação para o futuro; um padrão, consistência de um comportamento ao longo do tempo; perspectiva, maneira fundamental da organização de realizar as coisas; e também como truque, uma manobra específica para enganar o concorrente. Visto que todas essas contextualizações cabem para o termo aqui aplicado, podemos inferir que essa diversificação influencia diretamente na predominância do mesmo nos textos analisados.

Revista Produção Online, Florianópolis, SC, v.15, n. 3, p. 1021-1048, jul./set. 2015. 
Modelo de negócio é a "lógica de como uma organização cria, distribui e captura valor" (Osterwalder; Pigneur, 2010, p.14). Esse termo está presente em $27 \%$ das publicações analisadas. Já Fenômeno é defino por Kant (1985) como aquilo que é objeto da experiência sensível. E Sistema, conforme Chiavenato (2003) é o conjunto de elementos interdependentes, cujo resultado final é maior do que a soma de seus resultados caso operassem de maneira isolada, aparecem em $11 \%$, ambos.

Negócio e Setor, também tiveram a mesma porcentagem, sendo adotados por $3 \%$ dos autores, enquanto Filosofia e Princípios estavam ambos, em 1,5\% dos trabalhos. A figura 5 expõe o resultado dessa analise, apresentando o termo e a quantidade de publicações que este foi citado.

Figura 5 - Termos utilizados para denominar fast-fashion

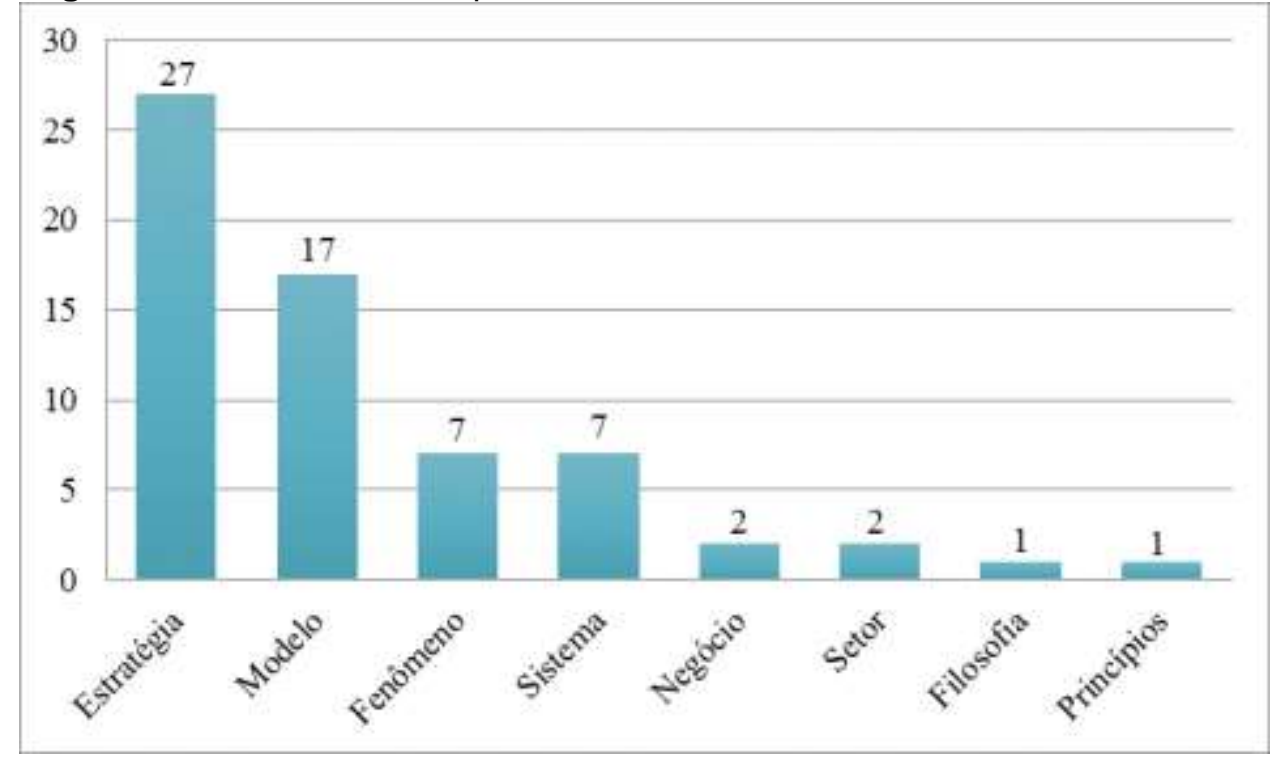

Fonte: Elaborado pelos autores (2014)

Apesar de Estratégia ter sido o termo mais citado, de acordo com o gráfico acima, entende-se que o mesmo é um conceito restrito e que limita as dimensões do fast-fashion, pois se constatou que este evoluiu ao longo do tempo e atualmente abrange toda a organização. Assim, o termo Estratégia não é adequado para denominar o FF. Modelo, por sua vez, foi o segundo termo com maior presença, apresentando uma definição mais consistente, que é reforçada por Cietta (2010), quando diz que o fast-fashion é um modelo presente em todos os setores de uma 
empresa, regendo amplamente suas atividades. Com base nesses argumentos, neste trabalho adotou-se o termo modelo para denominá-lo.

\section{AGENDA DE PESQUISA}

Identificaram-se algumas áreas dentro da temática fast-fashion que necessitam de um estudo mais aprofundado. Estes são brevemente apresentados, sendo eles: FF em países emergentes; FF em pequenas empresas; FF em outros setores; Obstáculos do FF.

\subsection{FF em países emergentes}

Nos países desenvolvidos o modelo está implantado e tem capacidade de funcionar, porém não existem muitos estudos que mostrem a aplicação em países emergentes. Esses países possuem como característica um setor industrial em desenvolvimento, o que muitas vezes significa que as tecnologias utilizadas não são de última geração. Doeringer e Crean (2006) alegam que com exceção do design, layout, e do corte, o resto da produção mudou pouco desde o século XIX, significando que nesses países, para a implantação do modelo, será necessário investir principalmente nesses três itens.

Contudo, segundo Rickman e Cosenza (2007), países como a China, Marrocos e Turquia, já possuem alta qualidade e flexibilidade na fabricação, sendo a qualidade também encontrada na mão-de-obra indiana e turca. Visto que muitas empresas aproveitam essa força de trabalho barata, porém distante dos seus mercados (Sull; Turconi, 2008), os países emergentes poderiam utilizar a sua mãode-obra própria e seu amplo mercado consumidor, como vantagem competitiva, aplicando o mesmo modelo. Entretanto, em alguns países desses países existem barreiras que dificultariam esta aplicação, tais como a carga tributária desfavorável.

\subsection{FF em pequenas empresas}

Apesar de o fast-fashion ser mais comumente estudado em grandes empresas, este modelo de negócios também pode ser aplicado em pequenas Revista Produção Online, Florianópolis, SC, v.15, n. 3, p. 1021-1048, jul./set. 2015. 
empresas de abrangência local. De acordo com Cietta (2010), o conhecimento do mercado é um dos pontos fortes das empresas FF. Além de trazer benefícios para a empresa que o adota, o FF também se torna muito eficaz ao defender o patrimônio produtivo de uma determinada região ou país, uma vez que consegue explorar a vantagem de saber antecipar as tendências de consumo locais, mais do que competidores globais.

A fim de incorporar este novo modelo de indústria, é necessário que as empresas modifiquem não só os métodos de design e marketing, como também as operações de negócios de forma completa, incluindo a atualização de tecnologias da informação e estratégias de compras (Peterson et al., 2010). Pequenas empresas geralmente não englobam em seus sistemas todas as fases do ciclo produtivo. Adquirir e desenvolver as competências necessárias para tornar o gerenciamento entre compradores e subfornecedores eficaz é a principal característica que pequenas empresas precisam desenvolver para utilizarem o modelo de forma satisfatória.

Além das vantagens descritas acima, os custos de transporte somam-se aos benefícios do uso do modelo para pequenas empresas. Para Arrigo (2010), a proximidade geográfica ajuda a tornar simples, do ponto de vista dos custos, o sistema logístico, o que incide diretamente no valor do produto final. Segundo Caro e Gallien (2010), embora a produção local aumente os custos referentes à mão de obra, ela fornece maior flexibilidade de oferta e maior receptividade do mercado.

Por a proximidade geográfica do mercado consumidor não ser um fator imitável por nenhum produtor estrangeiro de baixo custo, o fast-fashion se transforma em uma estratégia de política industrial bastante eficaz, uma vez que desenvolvê-lo permite defender a indústria nacional contra os impostos e outras barreiras do comércio internacional (Cietta, 2010).

\subsection{FF em outros setores}

Os seis autores considerados na tabela 6, apresentaram setores que possuem produtos com características fashion, e que por este motivo, têm condições de aplicar o FF. São eles: Arquitetura; Artes; Ciência; e os setores Alimentício, Automobilístico, e Tecnológico.

Revista Produção Online, Florianópolis, SC, v.15, n. 3, p. 1021-1048, jul./set. 2015. 
Tabela 6 - Modelo fast-fashion em outros setores

\begin{tabular}{ll}
\hline Setor & Autores \\
\hline Arquitetura & Rickman; Cosenza, 2007 \\
Artes & Rickman; Cosenza, 2007; Agripina, 2012 \\
Ciência & Agripina, 2012 \\
Setor Alimentício & Byun; Sternquist, 2008; Düffelmeyer, 2012 \\
Setor Automobilística & Díaz, 2005; Göranson; Hansson, 2010 \\
Setor Tecnológico & Díaz, 2005; Göranson; Hansson, 2010 \\
\hline
\end{tabular}

Fonte: Elaborada pelos autores (2014)

Estes setores foram citados como cabíveis de aplicação do FF por terem características semelhantes as do modelo em questão. A moda engloba todos os tipos de fenômenos culturais, como artes, arquitetura, e ciência, além de ser conceituada como processo de desenvolvimento social de dispersão pelo qual um novo estilo é adotado por alguns grupos de consumidores (Rickman; Cosenza, 2007; Agripina, 2012). O FF, de acordo com Byun e Sternquist (2008), é semelhante ao setor de alimentos e serviços no que concerne à perecibilidade. Os setores tecnológico e automobilístico aliam as características de moda e de vida útil curta, sendo desta maneira semelhante ao fast-fashion.

A partir do momento em que as empresas utilizarem seu potencial criativo e começarem a prever a tendência de mudança no mercado, o modelo FF poderá ser aplicado em outros setores de maneira positiva (Cietta, 2010). Essa expansão para outras industriais é ratificada por Suri (2002), que aponta os objetivos de desempenho do QRM, um modelo já utilizado por diversos setores, como sendo rapidez, custo e qualidade, análogos aos do FF.

\subsection{Obstáculos do FF}

Da mesma forma que qualquer outro modelo produtivo, o FF apresenta características que são consideradas desafios pelos autores, como pode ser visualizado na tabela 7. Entre os artigos que expuseram estas características, 9 deles consideraram que os produtos são descartáveis, seguido pela característica de ser insustentável (8), explorar a mão de obra (6) e promover o consumismo (4). 
Tabela 7 - Obstáculos do fast-fashion

\begin{tabular}{lc}
\multicolumn{1}{c}{ Nome } & № de citações \\
\hline Produtos descartáveis & 9 \\
Insustentável & 8 \\
Exploração da mão de obra & 6 \\
Consumismo & 4 \\
Experiência negativa do consumidor & 2 \\
Rouba mercado de empresas locais & 2 \\
Baixa qualidade & 1 \\
Exploração das condições locais & 1 \\
Formação de filas & 1 \\
Marcas de luxo reduzem seus padrões & 1 \\
\hline
\end{tabular}

Fonte: Elaborada pelos autores (2014)

Os produtos são considerados descartáveis por possuírem uma baixa qualidade (Bhardwaj; Fairhurst, 2010; Zarley, 2010; Cachon; Swinney, 2011; Fojtu, 2011; Hill, 2011; Kalinin, 2013), uma durabilidade limitada (Andersson; Molin, 2013; Kalinin, 2013), ou seja, um curto ciclo de vida (Fojtu, 2011; Hill, 2011; Forozin et al., 2012; Watson; Yan, 2013). Ao falar que o fast-fashion é insustentável, todos os autores apontam a geração de resíduos e o impacto negativo causado no meio ambiente. Por fim, pensando na exploração da mão de obra, os autores expõem os trabalhadores irregulares/infantil (Arrigo, 2013; Kim et al., 2013; Rossi, 2013), a questão das horas extras (Joy et al., 2012; KIM et al., 2013) e as condições antiéticas de trabalho (Reinach, 2005; Joy et al., 2012; Portway, 2012; Kim et al., 2013).

\section{CONSIDERAÇÕES FINAIS}

A literatura relevante para a temática fast-fashion foi classificada, sintetizada e analisada, utilizando o método de revisão bibliográfica sistemática. A revisão da literatura indica que há um crescente interesse por parte da academia na questão, uma vez que o volume de estudos sobre o FF vem crescendo com o passar dos anos.

Este trabalho contribuiu com seu extenso estudo sobre o estado da arte, o que envolve a área funcional considerada no estudo, a definição conceitual, o termo utilizado pelos pesquisadores, e os objetivos de desempenho no FF. Alguns autores abordam o fast-fashion como uma estratégia, outros como um modelo, um sistema, um fenômeno, entre outros. Nesse trabalho utilizamos o termo modelo uma vez que

Revista Produção Online, Florianópolis, SC, v.15, n. 3, p. 1021-1048, jul./set. 2015. 
o FF está presente em todos os setores de uma empresa e rege amplamente suas atividades.

A partir do estudo do estado da arte também foi possível definir o fast-fashion como um modelo de negócios que consegue unir a estratégia produtiva do quickresponse com a utilização de recursos avançados para o design de produtos, apresenta flexibilidade em todos os setores da cadeia produtiva para que possa otimizar o processo produtivo e atender com inovação a demanda dos consumidores que possuem gostos frequentemente mutáveis. Além de oferecer tendências atuais de maneira rápida e eficaz na forma de produtos com curto ciclo de vida e baixo custo.

A rapidez com que o consumidor tem acesso à informação provoca mudanças em seu comportamento, e isto está transformando o modelo de compra padronizada em uma maneira customizada que oferece produtos mais atrativos e com menor ciclo de vida. Esta mudança no comportamento não se limita ao setor têxtil, como consequência, empresas de outros setores da economia tenderão a adotar praticas do modelo FF.

Durante a revisão percebeu-se novos temas de pesquisa, como por exemplo, a análise conjunta da influência do Reino Unido, Suécia e Estados Unidos, uma vez que estes países se destacaram em diversos momentos do artigo. A agenda de pesquisa sugere ainda outros temas para estudos com o objetivo de buscar investigar como o fast-fashion se comporta em países emergentes, em pequenas empresas, e em outros setores industriais, além de compreender os obstáculos a serem ultrapassados pelas empresas que adotam o FF.

Entre os autores que tratam do tema FF, alguns apontam que a prática do fast-fashion leva a um maior uso de recursos naturais e ao consumismo excessivo. Dessa forma, um dos desafios das organizações FF será trabalhar com insumos que não agridam o meio ambiente, tais como materiais biodegradáveis, que gerem resíduos com menor impacto ao ambiente, e de fácil reciclagem, focando na sustentabilidade do planeta. Espera-se que esta revisão sirva como inspiração para estudos futuros sobre o fast-fashion, além de um guia para o assunto. 


\section{REFERÊNCIAS}

AGRIPINA, I. G. Scandinavian fashion brands: finding the puzzles between marketing strategy and Swedes customers behaviour. Dissertation, Universidad de Karlstad, Sweden, 2012.

ANDERSSON, S., MOLIN, J., A fast fashion company - to communicate environment and sustainability. Dissertation, Svensk Textilhögskolan, Sweden, 2013.

ARRIGO, E., Innovation and market-driven management in fast-fashion companies. Symphonya Emerging Issues in Management, v. 2, n. 1, p. 1-19, 2010. DOI: http://dx.doi.org/10.4468/2010.2.06arrigo

ARRIGO, E. Corporate responsibility management in fast fashion companies: the Gap Inc. case. Journal of Fashion marketing and management, v. 17, n. 2, p. 175-189, 2013. DOI: http://dx.doi.org/10.1108/JFMM-10-2011-0074

BARNES, L., LEA-GREENWOOD, G., Fast fashioning the supply chain: shaping the research agenda. Journal of Fashion Marketing and Management, v. 10, n. 3, p. 259-271, 2006. DOI: http://dx.doi.org/10.1108/13612020610679259

BARNES, L., LEA-GREENWOOD, G. Fast fashion in the retail store environment. International Journal of Retail \& Distribution Management, v. 38, n. 10, p. 760-772, 2010. DOI: http://dx.doi.org/10.1108/09590551011076533

BHARDWAJ, V., FAIRHURST, A. Fast fashion: response to changes in the fashion industry. The International Review of Retail, Distribution and Consumer Research, v. 20, n. 1, p. 165-173, 2010. DOI: http://dx.doi.org/10.1080/09593960903498300

BONNEFOI, T. Demand forecast for short life cycle products: Zara case study. Dissertation, Massachusetts Institute of Technology, USA, 2012.

BRANDTING, S., JOHANSSON, F. Labeling sustainability - can fast fashion become sustainable with the use of environmental and ethical labels? Dissertation, The Swedish School of Textiles, Sweden, 2012.

BRUCE, M., DALY, L.Buyer behaviour for fast fashion. Journal of Fashion Marketing and Management, v. 10, n. 3, p. 329-344, 2006. DOI: http://dx.doi.org/10.1108/13612020610679303

BRUCE, M., DALY, L. Adding value: challenges for UK apparel supply chain management - a review. Production Planning and Control, v. 22, n. 3, p. 210-220, 2011. DOI:

http://dx.doi.org/10.1080/09537287.2010.498574

BRUCE, M., DALY, L.; TOWERS, N. Lean or agile - a solution for supply chain management in the textiles and clothing industry. International Journal of Operations \& Production Management, v. 24, n. 2, p. 151-170, 2004. DOI: http://dx.doi.org/10.1108/01443570410514867

BYUN, S. E., STERNQUIST, B. The antecedents of in-store hoarding: measurement and application in the fast fashion retail environment. The International Review of Retail, 
Distribution and Consumer Research, v. 18, n. 2, p.133-147, 2008. DOI: http://dx.doi.org/10.1080/09593960701868241

CACHON, G. P., SWINNEY, R. The value of fast fashion: quick response, enhanced design, and strategic consumer behavior. Management Science, v. 57, n. 4, p. 778-795, 2011. DOI: http://dx.doi.org/10.1287/mnsc. 1100.1303

CAGLIANO, A. C., DEMARCO, A., RAFELE, C., VOLPE, S. Using system dynamics in warehouse management: a fast-fashion case study. Journal of Manufacturing Technology Management, v. 22, n. 2, p. 171-188, 2011. DOI:

http://dx.doi.org/10.1108/17410381111102207

CARO, F. C., GALLIEN, J., 2010. Inventory management of a fast-fashion retail network. Operations Research, v. 58, n. 2, p. 257-273, 2010. DOI:

http://dx.doi.org/10.1287/opre.1090.0698

CARO, F. C., GALLIEN, J. Clearance pricing optimization for a fast-fashion retailer. Operations research, v. 60 , n. 6 , p. 1404-1422, 2012. DOI:

http://dx.doi.org/10.1287/opre.1120.1102

CARO, F., MARTÍNEZ-DE-ALBÉNIZ, V., 2010. The impact of quick response in inventorybased competition. Manufacturing \& Service Operations Management, v. 12, n. 3, p. 409429, 2010. DOI: http://dx.doi.org/10.1287/msom.1090.0274

CARO, F., GALLIEN, J., DÍAZ, M., GARCÍA, J., CORREDOIRA, J. M., MONTES, M., RAMOS, J. A., CORREA, J. Zara uses operations research to reengineer its global distribution process. Interfaces, v. 40, n. 1, p. 71-84, 2010. DOI:

http://dx.doi.org/10.1098/rspb.2006.3551

CARRION, G. C., CACERES, K. T. Eco-shop paradox, a case study on Zara Rome. Dissertation, The Swedish School of Textiles, Sweden, 2012.

CHATVIJIT, S. Exploring the effects of scarcity, impulse buying, and product returning behavior in the fast fashion environment among female fashion conscious consumers. Dissertation, The University of North Carolina at Greensboro, USA, 2012.

CHENG, R. The desired-perceived identity gap of fast fashion retailers. Dissertation. The Manchester Metropolitan University, UK, 2010.

CHIAVENATO, I., 2003. Introdução à teoria geral da administração. Elsevier Brasil, 2003.

CHOI, T. M., CHIU, C. H., TO, K. M. C. A fast fashion safety-first inventory model. Textile Research Journal, v. 81, n. 8, p. 819-826, 2011. DOI:

http://dx.doi.org/10.1177/0040517510391697

CHOUDHURY, B., HOLMGREN, T., 2011. Supply chain agility towards global outsourcing within fast fashion industry, a case study at Gina Tricot. Dissertation, Jönköping University, Sweden, 2011.

CHRISTOPHER, M., LOWSON, R., PECK, H. Creating agile supply chains in the fashion industry. International Journal of Retail and Distribution Management, v. 32, n. 8, p. 367376, 2004. DOI: http://dx.doi.org/10.1108/09590550410546188 
CIETTA, E. A revolução do fast-fashion: estratégias e modelos organizados para competir nas indústrias híbridas. São Paulo: Estação das Letras e Cores, 2010.

COOK, D., Mulrow, C., Haynes, R. Systematic reviews: synthesis of best evidence for clinical decisions. Annals of Internal Medicine, v. 126, n. 1, p. 376-80, 1997.

CRESWELL, J. W. Projeto de Pesquisa: métodos qualitativo, quantitativo e misto. Trad. Luciana de Oliveira da Rocha. Porto Alegre: Artmed, 2007.

DÍAZ, F. C. An integrative framework for architecting supply chains. Dissertation, Massachusetts Institute of Technology, USA, 2005.

DOERINGER, P., CREAN, S. Can fast fashion save the US apparel industry?. Socioeconomic Review, v. 4, n. 3, p. 353-377, 2006. DOI: http://dx.doi.org/10.1093/ser/mwl014

DÜFFELMEYER, F. Nobody likes it, everybody buys it?! - the attitude-behavior gap in fast fashion. Dissertation, The Swedish School of Textiles, Sweden, 2012.

FERNIE, J. "Retail logistics", in Bruce, M., Moore, C. and Birtwistle, G. (Eds), International Retail Marketing, Butterworth-Heinemann, Oxford, p. 39-63, 2004. DOI:

http://dx.doi.org/10.1108/09590551011085975

FOJTU, M. Fast fashion and its global competitiveness. Dissertation, Univerzita Tomáše Bati ve Zlíve, Czech Republic, 2011.

FONSECA, F., ROZENFELD, H. Medição de desempenho para a gestão do ciclo de vida de produtos: uma revisão sistemática da literatura. Revista Produção Online, v. 12, n. 1, p. 159-184, 2012. DOI: http://dx.doi.org/10.14488/1676-1901.v12i1.853

FOROZIN, A., LUNDIN, C. I., SÖDERGREN, S. Appreciation of intrinsic value - in the context of fast fashion. Dissertation, The Swedish School of Textiles, Sweden, 2012.

GABRIELLI, V., BAGHI, I., CODELUPPI, V. Consumption practices of fast fashion products: a consumer-based approach. Journal of Fashion Marketing and Management, v. 17, n. 2, p. 206-224, 2013. DOI: http://dx.doi.org/10.1108/JFMM-10-2011-0076

GARRO, A. New product demand forecasting and distribution optimization: a case study at Zara. Dissertation, Massachusetts Institute of Technology, USA, 2011.

GONZÁLEZ, M., TOLEDO, J. A integração do cliente no processo de desenvolvimento de produto: revisão bibliográfica sistemática e temas para pesquisa. Revista Produção, v. 22, n. 1, p. 14-26, 2012. DOI: http://dx.doi.org/10.1590/S0103-65132011005000065

GÖRANSON, K., HANSSON, A. Fast fashion i modeindustrin: En kamp mot klockan. Dissertation, Linneuniversitetet - Ekonomihogskolan, Sweden, 2010.

HANSSON, M. What impact has a fast fashion strategy on fashion companies' supply chain management?. Dissertation, Halmstad University, Sweden, 2011.

HILL, J. T. Generation Y's perceptions of sustainable brand extensions of fast fashion retailers. Dissertation, The University of Texas at Austin, USA, 2011. 
HILLETOFTH, P., HILMOLA, O. P. Supply chain management in fashion and textile industry. International Journal of Services Sciences, v. 1, n. 2, p. 127-147, 2008. DOI: http://dx.doi.org/10.1504/IJSSCl.2008.019608

JOY, A., SHERRY, J. F., VENKATESH, A., WANG, J., CHAN, R., 2012. Fast fashion, sustainability, and the ethical appeal of luxury brands. Fashion Theory: The Journal of Dress, Body \& Culture, v. 16, n. 3, p. 273-296, 2012. DOI:

http://dx.doi.org/10.2752/175174112X13340749707123

KA YU, S., ZASTEZHKO, O. How can the Japanese specialty retailers of private-label apparel (SPAs) go into the German fast fashion market?. Dissertation, Mälardalen University, Sweden, 2008.

KALININ, D. Do fast fashion retailers have to be alert to changing customer values?-An examination of the material and meaning elements of the fast fashion practice. Dissertation, University of Gothenburg, Sweden, 2013.

KANT, I. Crítica da razão pura. São Paulo: Abril Cultural, 1985.

KIM, E. Y., PARK, K. Marketing mix elements influencing brand attitude strength: global vs. domestic SPA brands. Journal of Global Scholars of Marketing Science, v. 23, n. 3, p. 263281, 2013. DOI: http://dx.doi.org/10.1080/21639159.2013.788364

$\mathrm{KIM}, \mathrm{H} ., \mathrm{CHOO}, \mathrm{H}$. J., YOON, N. The motivational drivers of fast fashion avoidance. Journal of Fashion Marketing and Management, v. 17, n. 2, p. 243-260, 2013. DOI:

http://dx.doi.org/10.1108/JFMM-10-2011-0070

MADHANI, P. M. RFID deployment: fast fashion retailing. SCMS Journal of Indian Management, v. 1, n. 1, p. 40-51, 2011.

MEMIC, M., MINHAS, F. M. The fast fashion phenomenon. Dissertation, The Swedish School of Textiles, Sweden, 2011.

MILLER, K. Hedonic customer responses to fast fashion and replicas. Journal of Fashion Marketing and Management, v. 17, n. 2, p. 160-174, 2013. DOI:

http://dx.doi.org/10.1108/JFMM-10-2011-0072

MINTZBERG, H. Managing: desvendando o dia a dia da gestão. Porto Alegre: Bookman, 2010.

MOENG, R. Fast fashion retail: a consumer perspective. Dissertation, University of Pretoria, South Africa, 2012.

MURAN, L., Profile of H\&M: a pioneer of fast fashion. Textile Outlook International, July, p. 11-36, 2007.

OSTERWALDER, A., PIGNEUR, Y., 2010. Business model generation: inovação em modelos de negócios. New Jersey: Alta Books Editora, 2010.

PAI, M., MCCULLOCH, M., ENANORIA, W., COLFORD, J. M. Systematic reviews of diagnostic test evaluations: what's behind the scenes?. Evidence Based Medicine, v. 9, n. 4, p.101-103, 2004. DOI: http://dx.doi.org/10.1136/ebm.9.4.101 
PETERSON, J. H. A study of the dynamic capabilities along the fast fashion supply chain in China. Dissertation, The Hong Kong Polytechnic University, China, 2012.

PETERSON, J. H., CHANG, J., WONG, Y. H., LAWRENCE, C. A. The emergence of the fast fashion business model and imposed quick response challenges for Chinese fabric manufacturers. Innovative quick response programs in logistics and supply chain management, p. 387-422, 2010. DOI: http://dx.doi.org/10.1007/978-3-642-04313-0 20

PORTWAY, S. Regenerative abundance: fast and sustainable apparel production in Toronto. Dissertation, Ryerson University, Canada, 2012.

RÄISÄNEN, R. Agility in international fast fashion retailing. Dissertation, Turku School of Economics, Finland, 2013.

REINACH, S. S. China and Italy: fast fashion versus prêt à porter. Towards a new culture of fashion. Fashion Theory: The Journal of Dress, Body \& Culture, v. 9, n. 1, p. 43-56, 2005. DOI: http://dx.doi.org/10.2752/175174111X12954359478889

RICKMAN, T. A., COSENZA, R. M. The changing digital dynamics of multichannel marketing: the feasibility of the weblog: text mining approach for fast fashion trending. Journal of Fashion Marketing and Management, v. 11, n. 4, p. 604-621, 2007. DOI: http://dx.doi.org/10.1108/13612020710824634

ROSSI, A. Does economic upgrading lead to social upgrading in global production networks? Evidence from Morocco. World Development, v. 46, n. 1, p. 223-233, 2013. DOI: http://dx.doi.org/10.1016/j.worlddev.2013.02.002

ROWLEY, J., SLACK, F. Conducting a literature review. Management Research News, v. 27, n. 6, p. 31-39, 2004.. DOI: http://dx.doi.org/10.1108/01409170410784185

RUNFOLA, A., GUERCINI, S. Fast fashion companies coping with internationalization: driving the change or changing the model?. Journal of Fashion Marketing and Management, v. 17, n. 2, p. 190-205, 2013. DOI: http://dx.doi.org/10.1108/JFMM-10-2011$\underline{0075}$

SHERIDAN, M., MOORE, C., NOBBS, K. Fast fashion requires fast marketing: the role of category management in fast fashion positioning. Journal of Fashion Marketing and Management, v. 10, n. 3, p. 301-315, 2006. DOI:

http://dx.doi.org/10.1108/13612020610679286

SLACK, N., BRANDON-JONES, A., JOHNSTON, R. Princípios de administração da produção. Tradução: Ailton Bomfim Brandão. São Paulo: Editora Atlas S.A., 2013.

SULL, D., TURCONI, S. Fast-fashion lessons. Journal compilation - Business Strategy Review Summer, v. 19, n. 2, p. 4-11, 2008. DOI: http://dx.doi.org/10.1111/j.1467$\underline{8616.2008 .00527 . x}$

SURI, R. Quick response manufacturing: a competitive strategy for the 21 st century. In: Polca Implementations Workshop, p. 1-25, 2002.

TOKATLI, N. Global sourcing: insights from the global clothing industry - the case of Zara, a fast fashion retailer. Journal of Economic Geography, v. 8, n. 1, p. 21-38, 2008.. DOI: http://dx.doi.org/10.1093/jeg/lbm035

Revista Produção Online, Florianópolis, SC, v.15, n. 3, p. 1021-1048, jul./set. 2015. 
TOKATLI, N., KIZILGÜN, Ö., Cho, J. E. The clothing industry in Istanbul in the era of globalisation and fast fashion. Urban Studies, v. 48, n. 6, p. 1201-1215, 2010. DOI: http://dx.doi.org/10.1177/0042098010370629

WATSON, M. Z., YAN, R. N. An exploratory study of the decision processes of fast versus slow fashion consumers. Journal of Fashion Marketing and Management, v. 17, n. 2, p.141-159, 2013. DOI: http://dx.doi.org/10.1108/JFMM-02-2011-0045

WEBSTER, J., WATSON, R. T. Analyzing the past to prepare for the future: writing a literature review. Management Information Systems Quarterly, v. 26, n. 2, p. 3, 2002.

ZARLEY, M. An exploratory investigation of the decision processes and psychographic characteristics of fast versus slow fashion consumers. Dissertation, Colorado State University, USA, 2010.

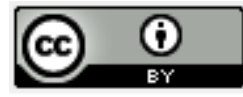

Artigo recebido em 07/12/2014 e aceito para publicação em 11/12/2014

DOI: http://dx.doi.org/ 10.14488/1676-1901.v15i3.1947 